Research Paper

\title{
A Low Lymphocyte-to-Monocyte Ratio Predicts Unfavorable Prognosis in Pathological T3N0 Rectal Cancer Patients Following Total Mesorectal Excision
}

\author{
Wei-Wei Xiao ${ }^{1^{*}}$, Lu-Ning Zhang ${ }^{1 *}$, Kai-Yun You ${ }^{2}$, Rong Huang ${ }^{1}$, Xin Yu ${ }^{1}$, Pei-Rong Ding ${ }^{3}$, Yuan-Hong \\ $\mathrm{Gao}^{1 凶}$ \\ 1. Department of Radiation Oncology, Sun Yat-sen University Cancer Center, State Key Laboratory of Oncology in South China, Collaborative \\ Innovation Center of Cancer Medicine, Guangzhou, China \\ 2. Department of Oncology, Sun Yat-Sen Memorial Hospital, Sun Yat-Sen University, Guangzhou, China \\ 3. Department of Colorectal Cancer, Sun Yat-sen University Cancer Center, State Key Laboratory of Oncology in South China, Collaborative Inno- \\ vation Center of Cancer Medicine, Guangzhou, China \\ * Equal contribution \\ $\square$ Corresponding author: YuanHong Gao, M.D., Department of Radiation Oncology, Sun Yat-sen University Cancer Center, State Key Laboratory of Oncology \\ in South China, Collaborative Innovation Center of Cancer Medicine, Guangzhou, China. Telephone: (86) 20-87343491; Fax: (86) 20-8734-3089; e-mail: \\ gaoyh@sysucc.org.cn
}

() 2015 Ivyspring International Publisher. Reproduction is permitted for personal, noncommercial use, provided that the article is in whole, unmodified, and properly cited. See http:/ /ivyspring.com/terms for terms and conditions.

Received: 2015.01.19; Accepted: 2015.04.15; Published: 2015.05.23

\begin{abstract}
Neoadjuvant radio-chemotherapy followed by total mesorectal excision (TME) is the standard treatment option for stage II and III rectal cancer. However, for PT3NO rectal cancer patients who receive upfront TME, the lack of an efficient method to predict their prognosis hampers postoperative treatment. A low lymphocyte-to-monocyte ratio (LMR) is associated with an unfavorable prognosis for certain malignancies; however, this association has not been investigated in rectal cancer. The purpose of this study was to evaluate whether LMR can predict the prognosis of PT3N0 rectal cancer patients following TME. Rectal cancer patients who received radical TME without preoperative treatment between June 2004 and Nov. 2011 at the Sun Yat-sen University Cancer Center were retrospectively reviewed. Counts for pre-surgery peripheral absolute lymphocytes and monocytes were obtained and used to calculate the LMR. A retrospective cohort of 280 PT3N0 rectal cancer patients who received TME was recruited. Significantly worse disease-free survival can be observed in patients with lower LMR levels $(<3.78)$ using univariate and multivariate analyses $(P=0.01$ and $P=0.015$, respectively). Subgroup analysis in patients with elevated carcinoembryonic antigen (CEA) and LMR $<3.78$ exhibited an accumulated 5 -year disease failure rate of approximately $40 \%$, whereas patients with normal CEA regardless of LMR and patients with LMR $\geq 3.78$ exhibited accumulated 5 -year disease failure rates of only approximately $15 \%$. Low pre-surgery peripheral LMR was significantly unfavorable for PT3N0 rectal cancer patient prognosis, especially in patients with elevated CEA. This easily obtained variable might serve as a valuable marker to predict the outcomes of PT3NO rectal cancer and indicate appropriate postoperative management.
\end{abstract}

Key words: Rectal cancer, Pathological T3N0, Lymphocyte-to-monocyte ratio, Total mesorectal excision

\section{Introduction}

Neoadjuvant chemoradiotherapy followed by total mesorectal excision (TME) has gradually been accepted as the standard treatment for patients with locally advanced rectal cancer. However, controversy remains regarding the peri-operative treatment strategy of patients with stage II rectal cancer, espe- 
cially in patients with pathological T3N0 (pT3N0) after upfront TME. Due to the varied prognosis of pT3N0 disease, it is critical to define receivable prognostic factors that may help these patients benefit from postoperative adjuvant therapy. Prognostic factors that have been identified in previous studies include carcinoembryonic antigen (CEA), vascular invasion, tumor size, and perineural invasion [1].

Emerging evidence indicates that cancer-associated inflammation plays a key role in the development and survival of a broad range of cancers [2]. Peripheral blood cells might reflect the inflammatory status of patients and the response of patients to malignant tumors, and these cells hold great promise for improving the predictive ability of known prognostic factors. Lymphocytes and monocytes are key immune cells in the inflammatory response and are independently associated with the prognosis of various malignancies, such as breast cancer [3], gastric cancer [4], hepatocellular carcinoma [5], acute lymphoblastic leukemia [6], and lymphoma [7].

Interestingly, a lower pretreatment lymphocyte-monocyte ratio (LMR) is associated with unfavorable prognosis in some hematology malignancies [8,9], although relevant studies in non-hematology malignancies have only been performed recently. Some studies have demonstrated a prognostic role for peripheral LMR at diagnosis in limited cancers, such as soft tissue sarcomas [10] and nasopharyngeal carcinoma [11].

To date, the prognostic value of the LMR in rectal cancer has not been reported. Therefore, we hypothesized that LMR might also play an important role in rectal cancer. The aim of this study was to investigate the prognostic value of baseline LMR for patients with pT3N0 rectal cancer. To our knowledge, this study is the first to examine the association between LMR and rectal cancer.

\section{Materials and Methods}

\section{Ethics statement}

This research was approved by the Ethical Committee of Sun Yat-sen University Cancer Center, and written informed consent was obtained from participants for the use of their clinical records in this study. The study complies with current Chinese law and was performed in accordance with the principles of Declaration of Helsinki.

\section{Patients}

We conducted a retrospective study on consecutive patients with rectal cancer who underwent curative TME at Sun Yat-sen University Cancer Center between June 2004 and Nov. 2011. The main inclusion criteria were as follows: pathologically proven pT3N0 rectal cancer, complete surgical resection, and no preoperative therapy. Patients were excluded if they died of postoperative complications or with positive margins.

\section{Evaluation and staging}

Clinical stage was assessed according to endorectal ultrasound, computed tomography (CT) and magnetic resonance imaging (MRI) examinations. In our cancer center, endorectal ultrasound is recommended for every patient because it provides accurate $\mathrm{T}$ staging. Abdominal CT and pelvic CT or MRI are examined routinely. Other tests, such as complete blood count (CBC) and liver function tests were also performed. Tumor stage was based on the 7th edition of the TNM Classification.

\section{Treatment}

Surgical resection was defined as radical when there was no evidence of distant metastases and tumor clearance was both macroscopically and histologically complete. All operations were conducted according to TME principles by colorectal surgeons, and the methods included low anterior resection (LAR) and abdominoperineal resection (AR).

\section{Laboratory data}

As part of pretreatment evaluation, peripheral blood was collected before any treatment, and peripheral lymphocytes and monocytes were counted using an automated hematology analyzer. The baseline peripheral LMR was calculated as the ratio of absolute counts between peripheral lymphocytes and monocytes. All patients had no self-reported acute infections, indicating that the cell counts represented a normal baseline value.

\section{Follow-up}

Follow up was performed every 3 months for the first 2 years after treatment and every 6 months thereafter. Evaluations included a $\mathrm{CBC}$, liver function test, serum levels of CEA and CA19-9, physical examination and digital rectal examination at each visit. Chest radiography, CT scanning of the abdomen and pelvis and colonoscopy were conducted every 6 months after surgery. PET/CT is not regularly recommended, although some patients preferred its advantages regarding the early detection of recurrence. Each patient follow-up appointment was recorded in a database.

\section{Statistical analysis}

The primary endpoint of the present study was disease-free survival (DFS), which was defined as the time between operation and failure (including recurrence and/or distant metastasis). All statistical anal- 
yses were performed using SPSS software, version 19.0 (SPSS Inc., Chicago, IL, USA). The distributions of LMR were compared using the chi-square test or Fisher's exact test. DFS was analyzed and compared using the Kaplan-Meier method and the log-rank test. Multivariate analysis was performed using Cox proportional hazards regression, and all possible clinical factors were entered in the analysis. A two-tailed $\mathrm{P}$ value of $<0.05$ was considered significant.

\section{Results}

\section{Patient characteristics}

In our study, 280 patients were enrolled; the median follow-up period for all patients was 52 months (range, 0.5-106.37 months). The basic clinicopathological characteristics of the patients are presented in Table 1. Fifty patients (17.8\%) experienced disease recurrence. Thirty-two patients $(11.4 \%)$ died after the last follow-up.

Absolute lymphocyte count (ALC) and absolute monocyte count (AMC) at time of initial diagnosis were derived from $C B C$ counts. The distribution of the LMR is shown in Figure 1. The median value of LMR was 3.78, with an inter-quartile range of 2.73-4.82.

\section{Correlations between LMR and clinicopatho- logical factors}

The correlations between LMR and clinicopathological factors are shown in Table 1. Using the median value of LMR (3.78) for the patient group, significant correlations were observed between LMR and sex $(\mathrm{P}=0.001)$ and between LMR and operation type $(\mathrm{P}=0.047)$. No significant correlation was observed between LMR and CEA level.

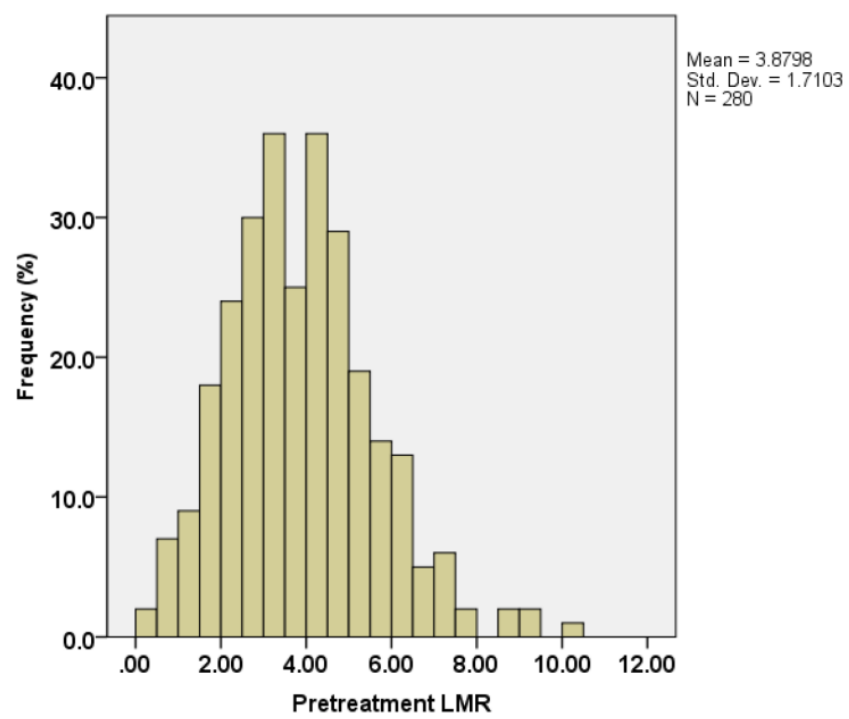

Figure 1. Distribution of lymphocyte-to-monocyte ratios (LMRs) in 280 PT3NOMO rectal cancer patients.
Table 1. Characteristics of 280 patients with pathological T3N0 rectal cancer according to LMR

\begin{tabular}{|c|c|c|c|c|c|}
\hline Characteristic & Overall & LMR $<3.78$ & LMR $\geq 3.78$ & Chi-Square & P value \\
\hline \multicolumn{6}{|l|}{ Gender } \\
\hline Male & 175 & 101 & 74 & 11.109 & 0.001 \\
\hline Female & 105 & 39 & 66 & & \\
\hline \multicolumn{6}{|l|}{ Age, y } \\
\hline$<61$ & 133 & 69 & 64 & 0.358 & 0.550 \\
\hline$\geq 61$ & 147 & 71 & 76 & & \\
\hline \multicolumn{6}{|l|}{ CEA level } \\
\hline Normal & 163 & 78 & 85 & 0.719 & 0.396 \\
\hline Elevated & 117 & 62 & 55 & & \\
\hline \multicolumn{6}{|l|}{ Tumor location } \\
\hline$\leq 5 \mathrm{~cm}$ & 73 & 35 & 38 & 0.167 & 0.683 \\
\hline$>5 \mathrm{~cm}$ & 207 & 105 & 102 & & \\
\hline \multicolumn{6}{|l|}{ Operation } \\
\hline AR & 227 & 107 & 120 & 3.933 & 0.047 \\
\hline APR & 53 & 33 & 20 & & \\
\hline \multicolumn{6}{|l|}{ Tumor grade } \\
\hline I & 19 & 11 & 8 & 1.205 & 0.547 \\
\hline II & 239 & 120 & 119 & & \\
\hline III & 22 & 9 & 13 & & \\
\hline \multicolumn{6}{|c|}{ Adjuvant treatment } \\
\hline No & 84 & 43 & 41 & 0.068 & 0.794 \\
\hline Chemotherapy & 196 & 97 & 99 & & \\
\hline
\end{tabular}

\section{Survival analyses}

As shown in Table 2, the correlation between DFS and each clinicopathological variable was examined using univariate analysis; normal CEA level (hazard ratio $[\mathrm{HR}]=0.470 ; 95 \% \mathrm{CI}, 0.266-0.830 ; \mathrm{P}=$ 0.009), higher LMR (as a continuous variable) (HR=0.790; 95\%CI, 0.661-0.945; $\mathrm{P}=0.010)$ and female gender (HR=0.506; 95\% CI, 0.264-0.970; $\mathrm{P}=0.040)$ were associated with a significantly lower risk of disease recurrence.

Table 2. Univariate and multivariate analyses of LMR for DFS in patients with $\mathrm{PT} 3 \mathrm{~N} 0 \mathrm{MO}$ rectal cancer

\begin{tabular}{|c|c|c|c|c|}
\hline & Univariate analysis & & Multivariate & analysis \\
\hline Variable & HR $(95 \%$ CI $)$ & P value & HR $(95 \% \mathrm{CI})$ & $P$ value \\
\hline CEA level & $0.470(0.266-0.830)$ & 0.009 & $\begin{array}{l}0.508 \\
(0.287-0.900)\end{array}$ & 0.021 \\
\hline LMR & $0.790(0.661-0.945)$ & 0.010 & $\begin{array}{l}0.805 \\
(0.675-0.959)\end{array}$ & 0.015 \\
\hline Gender & $0.506(0.264-0.970)$ & 0.040 & & \\
\hline Age & $1.026(1.000-1.053)$ & 0.052 & & \\
\hline Operation & $0.760(0.356-1.621)$ & 0.477 & & \\
\hline Tumor location & $0.731(0.402-1.327)$ & 0.303 & & \\
\hline Tumor grade & $0.939(0.463-1.903)$ & 0.862 & & \\
\hline Adjuvant treatment & $0.987(0.531-1.835)$ & 0.967 & & \\
\hline \multicolumn{5}{|c|}{$\begin{array}{l}\text { Bold values are significant }(\mathrm{P}<0.05) \text {. DFS, disease-free survival; HR, hazard ratio; } \\
\mathrm{CI} \text {, confidence interval }\end{array}$} \\
\hline \multicolumn{5}{|c|}{$\begin{array}{l}\text { The following parameters were included in the Cox proportional hazards model by } \\
\text { backward elimination: LMR as a continuous variable, CEA level (elevated vs. } \\
\text { normal), age as a continuous variable, gender (male vs. female), operation (APR vs. } \\
\text { AR), tumor location (low rectal cancer vs. high rectal cancer), tumor grade (highly } \\
\text { differentiated vs. moderately differentiated vs. poorly differentiated), and adjuvant } \\
\text { treatment (adjuvant chemotherapy vs. no chemotherapy). }\end{array}$} \\
\hline
\end{tabular}


When DFS was compared between patients with higher and lower LMR ( $\geq 2.73$ vs. $<2.73$; $\geq 3.78$ vs. $<3.78 ; \geq 4.82$ vs. $<4.82$ ), a significance difference was detected regardless of the cutoff point used (respective 5-year DFS rates: $83.6 \%$ vs. $70.6 \%, \mathrm{P}=0.009 ; 86.5 \%$ vs. $73.8 \%, \mathrm{P}=0.005$; and $88.5 \%$ vs. $77.2 \%, \mathrm{P}=0.041$; Figure 2).

Using multivariate analysis, CEA level $(\mathrm{HR}=0.508 ; 95 \% \mathrm{CI}, 0.287-0.900 ; \mathrm{P}=0.021)$ and LMR $(\mathrm{HR}=0.805 ; 95 \% \mathrm{CI}, 0.675-0.959 ; \mathrm{P}=0.015)$ remained significantly associated with DFS.

Because the CEA level was not significantly correlated with LMR and both of these factors were significantly associated with DFS in patients with pT3N0 rectal cancer, the patients were divided into four subgroups according to CEA level (elevated vs. normal) and LMR ( $\geq 3.78$ vs. $<3.78)$. As shown in Figure $3,22 \%$ of the patients with pT3N0 exhibited both elevated CEA and lower LMR before treatment; these patients exhibited lower DFS rates than the other three subgroups (Table 3). The 5-year accumulated disease recurrence rate was approximately $40 \%$ for patients with elevated CEA and lower LMR. In contrast, it was approximately $15 \%$ for the other three groups.

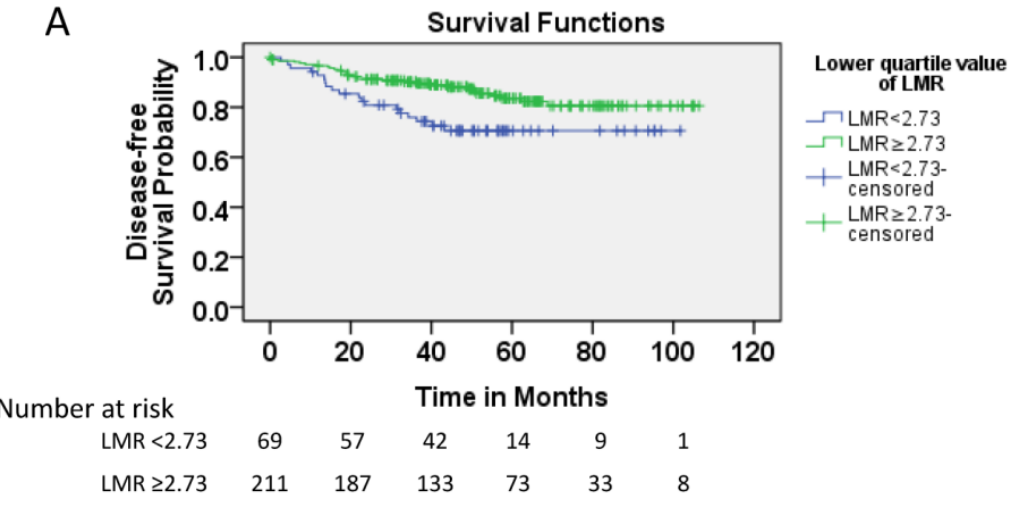

B
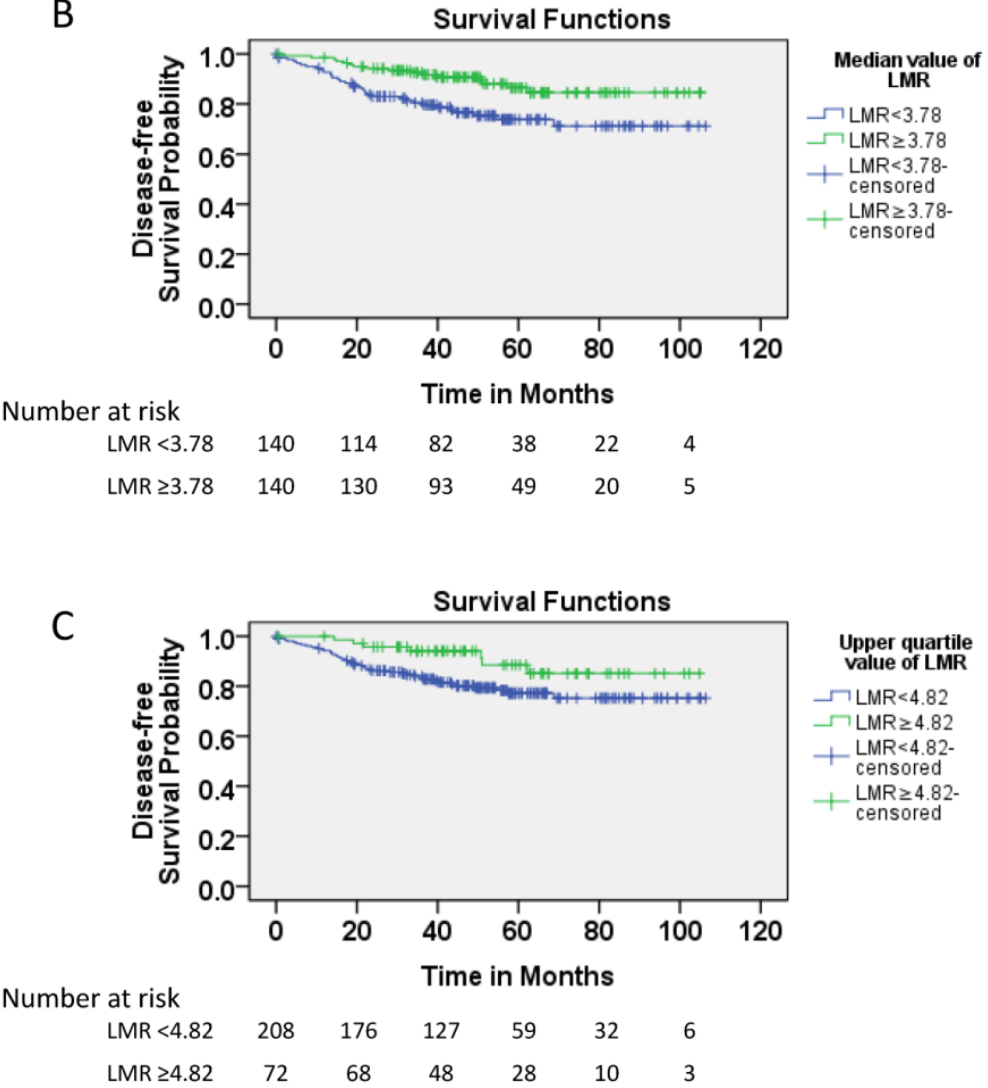

Figure 2. Elevated LMR indicates a favorable disease-free survival (DFS) in pT3NOMO rectal cancer patients. A. Cumulative DFS curves of patients with PT3NOMO rectal cancer with high or low LMR based on the median value (number of patients, 280; number of events, 49). B. Cumulative DFS curves of patients with PT3NOMO rectal cancer with high or low LMR based on the lower quartile value (number of patients, 280; number of events, 49). C. Cumulative DFS curves of patients with PT3NOMO rectal cancer with high or low LMR based on the upper quartile value (number of patients, 280; number of events, 49). 


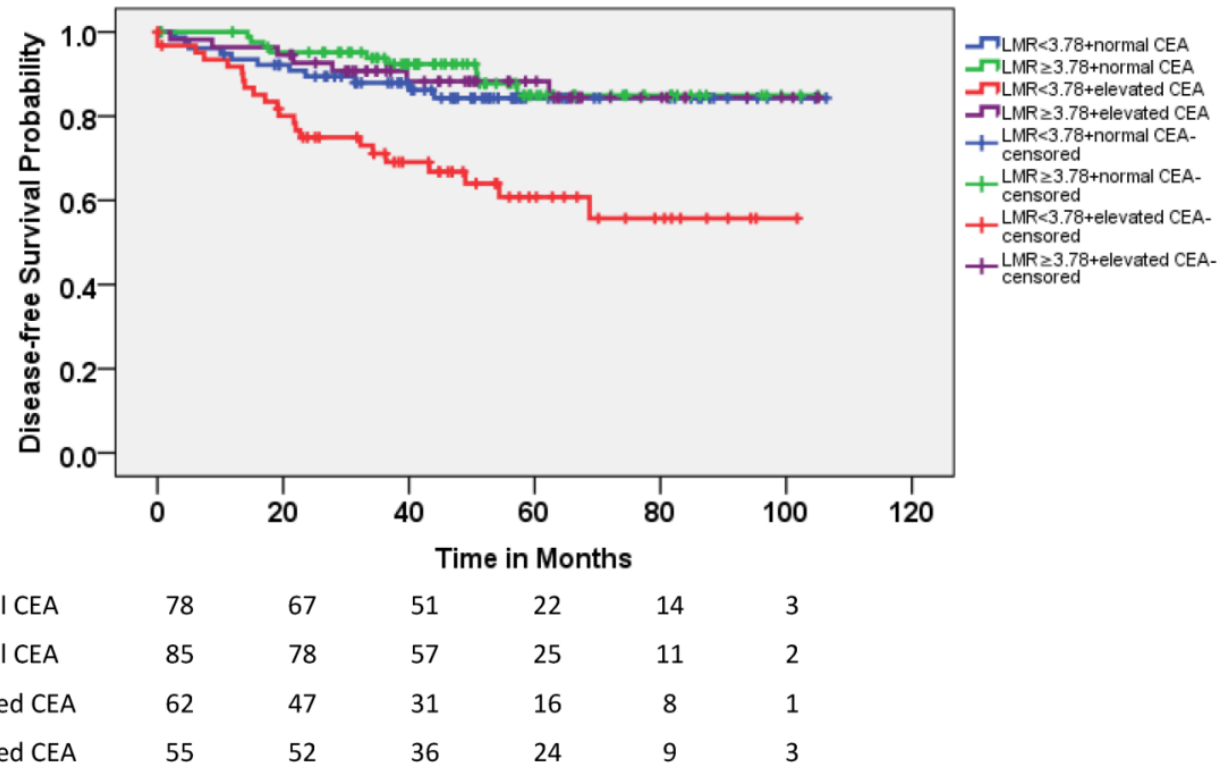

Figure 3. Kaplan-Meier estimates of DFS according to LMR and carcinoembryonic antigen (CEA) level.

Table 3. Comparison of DFS in patients with $\mathrm{PT} 3 \mathrm{~N} 0$ rectal cancer according to LMR and CEA

\begin{tabular}{|c|c|c|c|c|c|c|c|}
\hline & Subgroup & $\begin{array}{l}\text { LMR }<3.78+\text { normal } \\
\text { CEA }\end{array}$ & $\begin{array}{l}\text { LMR } \geq 3.78+\text { normal } \\
\text { CEA }\end{array}$ & $\begin{array}{l}\text { LMR }<3.78+\text { elevated } \\
\text { CEA }\end{array}$ & $\begin{array}{l}\text { LMR } \geq 3.78+\text { elevated } \\
\text { CEA }\end{array}$ & Chi-Square & $P$ value \\
\hline $\mathrm{N}(\%)$ & & $78(27.9)$ & $85(30.4)$ & $62(22.1)$ & $55(19.6)$ & 20.303 & $<0.001$ \\
\hline \multirow{5}{*}{ 5-year DFS } & & $84.3 \%$ & $84.9 \%$ & $60.8 \%$ & $88.3 \%$ & & \\
\hline & LMR $<3.78+$ normal CEA & - & - & - & - & & \\
\hline & LMR $\geq 3.78+$ normal CEA & $\begin{array}{l}\chi^{2}=0.569 \\
P=0.451\end{array}$ & - & - & - & & \\
\hline & LMR $<3.78+$ elevated CEA & $\begin{array}{l}\chi^{2}=8.486 \\
P=0.004\end{array}$ & $\begin{array}{l}X^{2}=14.237 \\
P<0.001\end{array}$ & - & - & & \\
\hline & LMR $\geq 3.78+$ elevated CEA & $\begin{array}{l}X^{2}=0.122 \\
P=0.727\end{array}$ & $\begin{array}{l}\chi^{2}=0.074 \\
P=0.785\end{array}$ & $\begin{array}{l}\chi^{2}=8.676 \\
P=0.003\end{array}$ & - & & \\
\hline
\end{tabular}

Bold values are significant $(\mathrm{P}<0.05)$

\section{Discussion}

Current guidelines issued by the National Comprehensive Cancer Network recommend that all patients with clinical stage II/III rectal cancer should be treated with preoperative radio-chemotherapy followed by TME. However, whether patients with pT3N0 rectal cancer who received upfront TME should undergo adjuvant therapy remains controversial.

An increasing number of studies suggest a strong link between inflammation and cancer, and the pretreatment of peripheral inflammatory cells, including neutrophils, lymphocytes and monocytes, has been significantly associated with progression and prognosis in various types of cancers.

The peripheral blood lymphocyte count is an important surrogate marker of immunological reconstitution following stem cell transplantation in non-Hodgkin lymphoma, and lymphopenia is a surrogate marker of host immunological incompetence
[9]. Lymphopenia prior to the initiation of systemic treatment is a powerful predictor of clinical outcome in hematologic and solid malignancies [12]. Lymphopenia prior to the initiation of systemic treatment is also a poor-risk feature in patients with stage II and III rectal cancer who have been treated with neoadjuvant chemoradiotherapy [13-15]. The induction of programmed cell death, antibody-dependent cellular cytotoxicity, and complement-dependent cytotoxicity play important roles in slowing or preventing disease progression and distant metastasis in rectal cancer [16]. Lymphopenia might impair the efficacy of the immune system by impairing antibody-dependent cell-mediated cytotoxicity due to a lack of effector cells.

Monocyte-associated macrophages might contribute to the suppression of host anti-tumor immunity and the promotion of tumor angiogenesis [17]. Furthermore, monocytes might also provide trophic factors that directly promote the growth and survival of cancer cells [18]. Wilcox et al. have shown that pe- 
ripheral blood monocytes and their progeny within the tumor microenvironment express the T-cell co-inhibitory ligand B7-H1 (PD-L1), which stimulates the expansion of suppressive regulatory $\mathrm{T}$ cells [19]. Monocytes are direct precursors of hematopoietic stem cell-derived macrophages. After recruitment into the tumor tissue, monocytes can differentiate into tumor-associated macrophages, which are an important component of infiltrating inflammatory cells, and might interact with tumor cells to promote tumor development by producing various cytokines and chemokines [20]. Therefore, it is not surprising that peripheral blood monocytosis is an adverse prognostic factor for various tumors [21-25].

In hematology malignancies $[8,21]$ and solid tumors [10,11,26-32], including lung cancer, bladder cancer, breast cancer, oropharyngeal cancer, pancreatic adenocarcinoma, gastric cancer and renal cell carcinoma, literature reports show that a low pre-treatment peripheral LMR level is significantly unfavorable and represents a useful marker for predicting outcomes.

To our knowledge, this is the first large-scale study to evaluate the prognostic significance of LMR in patients with rectal cancer and pT3N0 rectal cancer in particular. We performed a retrospective cohort study on 280 patients with pT3N0 rectal cancer who had received TME with or without adjuvant chemotherapy to evaluate the prognostic values of pretreatment peripheral LMR and other clinical factors. Our results confirmed previous findings that normal CEA levels were associated with a favorable prognosis for patients with rectal cancer [33]. More importantly, we found that a lower LMR was significantly associated with a high probability of disease failure and inferior DFS and was able to predict patient prognosis for pT3N0 rectal cancer after TME independently of other variables.

Interestingly, we also found that LMR was not significantly associated with CEA level. Moreover, LMR $<3.78$ was associated with inferior DFS in patients with elevated CEA but was not associated with a survival difference in patients with normal CEA. These results suggest that host immune status affects survival in patients with pT3N0, especially in patients with elevated CEA. Patients with rectal cancer who are staged as PT3N0 with elevated CEA level might already have micrometastases and are more likely to relapse after curative treatment. In those patients, an immune reaction, indicated by elevated LMR, might be sufficient to eradicate the micrometastases and afford good disease control, as with patients exhibiting normal CEA. Thus, in patients with both elevated CEA and LMR $<3.78$ at diagnosis, adjuvant radio-chemotherapy appears strongly recommended. In patients with normal CEA or LMR $\geq 3.78$, radical surgery might suffice, and postoperative adjuvant treatment might not lead to improved treatment outcomes.

\section{Conclusions}

Taken together, these findings show for the first time that pre-surgery low peripheral blood LMR predicts unfavorable prognosis in patients with pT3N0 rectal cancer, especially in patients with elevated CEA at initial diagnosis. This biomarker can be derived directly from routine blood cell counts and can easily be applied as a prognostic marker and as an individual treatment index for pT3N0 rectal cancer in the clinical setting. Further studies in a multicenter or prospective manner are warranted.

\section{Abbreviations}

TME: total mesorectal excision; pT3N0: pathological T3N0; CEA: carcinoembryonic antigen; LMR: lymphocyte-monocyte ratio; CT: computed tomography; MRI: magnetic resonance imaging; LAR: low anterior resection; AR: abdominoperineal resection; DFS: disease-free survival; CBC: complete blood count; HR: hazard ratio; ALC: absolute lymphocyte count; AMC: absolute monocyte count

\section{Acknowledgments}

This study was supported by the National Natural Science Foundation of China (No. 81071891, 81402533), the Science and Technology Planning Project of Guangdong Province, China (No. 2010B0807017), the Natural Science Foundation of Guangdong Province, China (No. S2013040013158), and the Young Scientists fund of Sun Yat-sen University, China (No. 12ykpy57).

\section{Competing interests}

The authors declare no competing interest.

\section{References}

1. Willett CG, Badizadegan K, Ancukiewicz M, Shellito PC. Prognostic factors in stage T3N0 rectal cancer: do all patients require postoperative pelvic irradiation and chemotherapy? Dis Colon Rectum. 1999;42:167-73.

2. Hanahan D, Weinberg RA. Hallmarks of cancer: the next generation. Cell. 2014;44:646-74.

3. Hornychova $\mathrm{H}$, Melichar B, Tomsova M, et al. Tumor-infiltrating lymphocytes predict response to neoadjuvant chemotherapy in patients with breast carcinoma. Cancer Invest. 2008;26:1024-31.

4. Bruckner HW, Lavin PT, Plaxe SC, et al. Absolute granulocyte, lymphocyte, and moncyte counts. Useful determinants of prognosis for patients with metastatic cancer of the stomach. JAMA. 1982;247:1004-6.

5. Chew V, Chen J, Lee D, et al. Chemokine-driven lymphocyte infiltration: an early intratumoural event determining long-term survival in resectable hepatocellular carcinoma. Gut. 2012;61:427-38.

6. Sun D, Elson P, Liedtke M, et al. Absolute lymphocyte count at day 28 independently predicts event-free and overall survival in adults with newly diagnosed acute lymphoblastic leukemia. Am J Hematol. 2012; 87: 957-960.

7. Wilcox RA, Ristow K, Habermann TM, et al. The absolute monocyte and lymphocyte prognostic score predicts survival and identifies high-risk patients in diffuse large-B-cell lymphoma. Leukemia. 2011; 25:1502-1509. 
8. Li YL, Pan YY, Jiao Y, et al. Peripheral blood lymphocyte/monocyte ratio predicts outcome for patients with diffuse large B cell lymphoma after standard first-line regimens. Ann Hematol. 2014;93:617-26.

9. Porrata LF, Ristow K, Colgan JP, et al. Peripheral blood lymphocyte/monocyte ratio at diagnosis and survival in classical Hodgkin's lymphoma. Haematologica. 2012; 97:262-9.

10. Szkandera J, Gerger A, Liegl-Atzwanger B, et al. The lymphocyte/monocyte ratio predicts poor clinical outcome and improves the predictive accuracy in patients with soft tissue sarcomas. Int J Cancer. 2014;135:362-70.

11. Li J, Jiang R, Liu WS, et al. A large cohort study reveals the association of elevated peripheral blood lymphocyte-to-monocyte ratio with favorable prognosis in nasopharyngeal carcinoma. PLoS One. 2013;8: e83069.

12. Ray-Coquard I, Cropet C, Van Glabbeke M, et al. Lymphopenia as a prognostic factor for overall survival in advanced carcinomas, sarcomas and lymphomas. Cancer Res. 2009;69:5383-91.

13. Choi CH, Kim WD, Lee SJ, et al. Clinical predictive factors of pathologic tumor response after preoperative chemoradiotherapy in rectal cancer. Radiat Oncol J. 2012;30:99-107.

14. Kim IY, You SH, Kim YW. Neutrophil-lymphocyte ratio predicts pathologic tumor response and survival after preoperative chemoradiation for rectal cancer. BMC Surg. 2014;14:94.

15. Krauthamer M, Rouvinov K, Ariad S, et al. A study of inflammation-based predictors of tumor response to neoadjuvant chemoradiotherapy for locally advanced rectal cancer. Oncology. 2013;85:27-32.

16. Liljefors M, Nilsson B, Hjelm Skog AL, et al. Natural killer (NK) cell function is a strong prognostic factor in colorectal carcinoma patients treated with the monoclonal antibody 17-1A. Int J Cancer. 2003;105:717-23.

17. Dirkx AE, Oude Egbrink MG, Wagstaff J, et al. Monocyte/macrophage infiltration in tumors: modulators of angiogenesis. J Leukoc Biol. 2006;80:1183-96.

18. Wilcox RA, Wada DA, Ziesmer SC et al. Monocytes promote tumor cell survival in T-cell lymphoproliferative disorders and are impaired in their ability to differentiate into mature dendritic cells. Blood. 2009;114:2936-44.

19. Schmidt H, Bastholt L, Geertsen P, et al. Elevated neutrophil andmonocyte counts in peripheral blood are associated with poor survival in patients with metastaticmelanoma: a prognostic model. Br J Cancer. 2005;93:273-8.

20. Richards DM, Hettinger J, Feuerer M. Monocyt es and macrophages in cancer: development and functions. Cancer Microenviron. 2013; 6:179-91.

21. Koh YW, Shin SJ, Park C, et al. Absolute monocyte count predicts overall survival in mantle cell lymphomas: correlation with tumour-associated macrophages. Hematol Oncol. 2014;32:178-86.

22. Mo H, Shi Y, Han X, et al. Absolute monocyte count is a prognostic indicator in a patient with diffuse large B-cell lymphoma after autologous peripheral blood stem cell transplant. Leuk Lymphoma. 2015;56:515-7.

23. Porrata LF, Ristow K, Markovic SN. Absolute monocyte count at diagnosis and survival in mantle cell lymphoma. Br J Haematol. 2013;63:545-7.

24. Tadmor T, Bari A, Sacchi S, et al. Monocyte count at diagnosis is a prognostic parameter in diffuse large B-cell lymphoma: results from a large multicenter study involving 9 patients in the pre- and post-rituximab era. Haematologica. 2014:99:25-30.

25. Nitta H, Terui Y, Yokoyama M, et al. Absolute peripheral monocyte count at diagnosis predicts central nervous system relapse in diffuse large B-cell lymphoma. Haematologica. 2015;100:87-90.

26. Huang SH, Waldron JN, Milosevic M, et al. Prognostic value of pretreatment circulating neutrophils, monocytes, and lymphocytes in oropharyngeal cancer stratified by human papillomavirus status. Cancer. 2015;121:545-55.

27. Hu P, Shen H, Wang G, et al. Prognostic significance of systemic inflammation-based lymphocyte- monocyte ratio in patients with lung cancer: based on a large cohort study. PLoS One. 2014;9:e08062

28. Temraz S, Mukherji D, Farhat ZA, et al. Preoperative lymphocyte-to-monocyte ratio predicts clinical outcome in patients undergoing radical cystectomy for transitional cell carcinoma of the bladder: a retrospective analysis. BMC Urol. 2014; $4: 76$

29. Fujiwara $Y$, Misawa T, Shiba H, et al. Postoperative peripheral absolute blood lymphocyte-to-monocyte ratio predicts therapeutic outcome after pancreatic resection in patients with pancreatic adenocarcinoma. Anticancer Res. 2014:34:563-8.

30. Zhou $\mathrm{X}, \mathrm{Du} \mathrm{Y}, \mathrm{Xu}$ J, et al. The preoperative lymphocyte to monocyte ratio predicts clinical outcomes in patients with stage II/III gastric cancer. Tumour Biol. 2014;35:11659-66.

31. Lin GN, Peng JW, Liu DY, et al. Increased lymphocyte to monocyte ratio is associated with better prognosis in patients with newly diagnosed metastatic nasopharyngeal carcinoma receiving chemotherapy. Tumour Biol. 2014; 35:10849-54

32. Hutterer GC, Stoeckigt C, Stojakovic T, et al. Low preoperative lymphocyte-monocyte ratio (LMR) represents a potentially poor prognostic factor in nonmetastatic clear cell renal cell carcinoma. Urol Oncol. 2014;32:1041-8.

33. Nissan A, Stojadinovic A, Shia J, et al: Predictors of recurrence in patients with $\mathrm{T} 2$ and early T3, N0 adenocarcinoma of the rectum treated by surgery alone. J Clin Oncol. 2006; 24:4078-84 